\begin{tabular}{|c|c|}
\hline Title & $\begin{array}{l}\text { Co thickness dependence of structural and magnetic properties in spin quantum cross devices utilizing stray magnetic } \\
\text { fields }\end{array}$ \\
\hline Author(s) & Kaiju, Hideo; Kasa, Haruy a; Komine, Takashi; Mori, Sumito; Misawa, Takahiro; A be, Taro; Nishii, Junji \\
\hline Citation & $\begin{array}{l}\text { Journal of applied physics, 117(17), 17C 738-1-17C738-4 } \\
\text { https://doi.org/10.1063/1.4917061 }\end{array}$ \\
\hline Issue Date & 2015-05-08 \\
\hline Doc URL & http:/hdl.handle.net/2115/59519 \\
\hline Rights & $\begin{array}{l}\text { Copyright } 2015 \text { A merican Institute of Physics. This article may be downloaded for personal use only. A ny other use } \\
\text { requires prior permission of the author and the A merican Institute of Physics. The following article appeared in J. A ppl. } \\
\text { Phys. } 117,17 C 738 \text { (2015) and may be found at http://dx.doi.org/10.1063/1.4917061. }\end{array}$ \\
\hline Type & article \\
\hline File Information & 1.4917061.pdf \\
\hline
\end{tabular}

Instructions for use 


\section{AIP hameded inges}

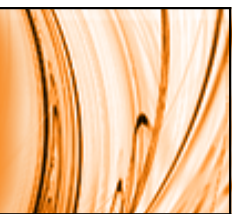

\section{Co thickness dependence of structural and magnetic properties in spin quantum cross devices utilizing stray magnetic fields}

H. Kaiju, H. Kasa, T. Komine, S. Mori, T. Misawa, T. Abe, and J. Nishii

Citation: Journal of Applied Physics 117, 17 C738 (2015); doi: 10.1063/1.4917061

View online: http://dx.doi.org/10.1063/1.4917061

View Table of Contents: http://scitation.aip.org/content/aip/journal/jap/117/17?ver=pdfcov

Published by the AIP Publishing

\section{Articles you may be interested in}

Magnetic properties of perpendicular exchange coupled composite with a synthesis antiferromagnetic sandwich structure

Appl. Phys. Lett. 104, 102402 (2014); 10.1063/1.4867904

Thickness dependence of magnetic and structural properties in Fe $80 \mathrm{Ga} 20$ thin films

J. Appl. Phys. 107, 09A944 (2010); 10.1063/1.3368107

Fabrication and properties of FePt thick films for alternative local field micromagnet

J. Appl. Phys. 105, 07A744 (2009); 10.1063/1.3076055

Micromagnetic simulation of magnetization reversal process and stray field behavior in Fe thin film wire J. Appl. Phys. 102, 123908 (2007); 10.1063/1.2821731

Low resistance spin-dependent magnetic tunnel junction with high breakdown voltage for current-inducedmagnetization-switching devices

J. Appl. Phys. 97, 10C926 (2005); 10.1063/1.1857651

Frustrated by

old technology?

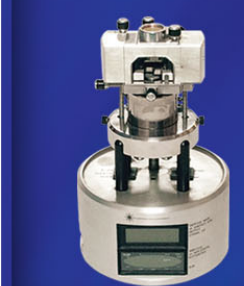

Is your AFM dead

and can't be repaired?

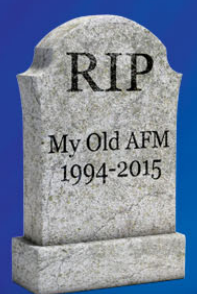

Sick of bad customer support?

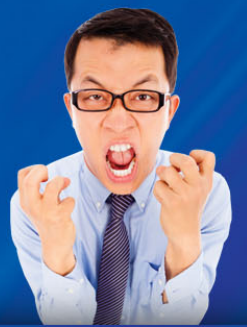

It is time to upgrade your AFM

Minimum $\$ 20,000$ trade-in discount for purchases before August 31st

Asylum Research is today's technology leader in AFM 


\title{
Co thickness dependence of structural and magnetic properties in spin quantum cross devices utilizing stray magnetic fields
}

\author{
H. Kaiju, ${ }^{1, a)}$ H. Kasa, ${ }^{1}$ T. Komine,${ }^{2}$ S. Mori, ${ }^{1}$ T. Misawa, ${ }^{1}$ T. Abe, ${ }^{1}$ and J. Nishii ${ }^{1}$ \\ ${ }^{1}$ Research Institute for Electronic Science, Hokkaido University, Sapporo, Hokkaido 001-0020, Japan \\ ${ }^{2}$ Faculty of Engineering, Ibaraki University, Hitachi, Ibaraki 316-8511, Japan
}

(Presented 7 November 2014; received 18 September 2014; accepted 3 December 2014; published online 7 April 2015)

\begin{abstract}
We investigate the Co thickness dependence of the structural and magnetic properties of Co thinfilm electrodes sandwiched between borate glasses in spin quantum cross (SQC) devices that utilize stray magnetic fields. We also calculate the Co thickness dependence of the stray field between the two edges of Co thin-film electrodes in SQC devices using micromagnetic simulation. The surface roughness of Co thin films with a thickness of less than $20 \mathrm{~nm}$ on borate glasses is shown to be as small as $0.18 \mathrm{~nm}$, at the same scanning scale as the Co film thickness, and the squareness of the hysteresis loop is shown to be as large as $0.96-1.0$. As a result of the establishment of polishing techniques for Co thin-film electrodes sandwiched between borate glasses, we successfully demonstrate the formation of smooth Co edges and the generation of stray magnetic fields from Co edges. Theoretical calculation reveals that a strong stray field beyond $6 \mathrm{kOe}$ is generated when the Co thickness is greater than $10 \mathrm{~nm}$ at a junction gap distance of $5 \mathrm{~nm}$. From these experimental and calculation results, it can be concluded that SQC devices with a Co thickness of 10-20 nm can be expected to function as spin-filter devices. (C) 2015 AIP Publishing LLC.
\end{abstract}

[http://dx.doi.org/10.1063/1.4917061]

\section{INTRODUCTION}

Spintronics is an emerging research field which utilizes the charge and spin degrees of freedom of electrons in solidstate systems. ${ }^{1-4}$ One of the fascinating phenomena in spintronics is the spin-filter effect, which allows us to obtain highly spin-polarized charge carriers in nonmagnetic materials. ${ }^{5-9}$ Recently, we proposed spin quantum cross (SQC) devices utilizing stray magnetic fields as a new type of spinfilter device. ${ }^{10}$ This SQC device consists of inorganic complexes or quantum dots (QDs) sandwiched between two crossed edges of magnetic thin-film electrodes. In this structure, a high magnetic field could be locally generated in the inorganic complexes or QDs due to the contributions of the stray field from both edges of the magnetic thin-film electrodes. Because a large magnetic field produces a large Zeeman effect, energy splitting of the inorganic complexes or QDs can be enhanced. Therefore, a large spin-filter effect can be expected. In our previous work, we reported some preliminary results for SQC devices. Typical results included the observation of ohmic characteristics in Ni/Ni devices, nanoscale tunneling phenomena in $\mathrm{Ni} / \mathrm{NiO} / \mathrm{Ni}$ devices, and the ballistic regime of nanoscale molecules in Ni/P3HT:PCBM/ Ni devices. ${ }^{11-13}$ Although such nanoscale phenomena have been observed in SQC devices, these devices cannot function as spin-filter devices, because the squareness of the hysteresis loop of magnetic thin films on polyethylene naphthalate (PEN) substrates, used in the magnetic electrodes of SQC devices, is not sufficiently large. ${ }^{14}$ In contrast, our ongoing research results suggest that Co thin films on borate glasses

${ }^{\text {a)} E l e c t r o n i c ~ m a i l: ~ k a i j u @ e s . h o k u d a i . a c . j p . ~}$ are good candidates for magnetic electrodes in SQC devices. However, the detailed structural and magnetic properties of such electrodes have not been clarified, especially in terms of their dependences on the Co film thickness. In this study, we investigate the dependences of the structural and magnetic properties of Co thin films on its thickness on borate glasses and Co thin-film electrodes sandwiched between borate glasses in SQC devices, and also calculate the dependence of the stray field between the two edges of Co thin-film electrodes on the Co film thickness in SQC devices using micromagnetic simulation.

\section{EXPERIMENTAL}

Co thin films were deposited on borate-glass substrates with a glass transition temperature $T_{\mathrm{g}}$ of $464^{\circ} \mathrm{C}$ (ISUZU GLASS CO., LTD.) under a magnetic field of 400 Oe by electron beam evaporation. The growth rate of the Co thin films was $0.15 \mathrm{~nm} / \mathrm{s}$ at an evaporation power of $160-200 \mathrm{~W}$. The dimensions of the borate-glass substrates were $10 \times 10 \times 2 \mathrm{~mm}^{3}$, and both sides were obliquely cut and polished before the deposition of the Co thin films. The Co thin films deposited on the borate-glass substrates were stacked on borate glasses of the same composition using a glass mold pressing technique at a glass deformation temperature $A_{\mathrm{t}}$ of $503^{\circ} \mathrm{C}$ under a pressure of $0.25 \mathrm{MPa}$ and then cut down to half of the samples. Finally, the cross-sectional surfaces of the stacked samples were polished by a mechanical polishing (MP) method using $\mathrm{Al}_{2} \mathrm{O}_{3}$-based emeries and a chemical mechanical polishing (CMP) method using $\mathrm{CeO}_{2}$ and $\mathrm{Al}_{2} \mathrm{O}_{3}$ slurries. The surface morphologies and roughnesses of the samples were analyzed by atomic force microscopy (AFM; 
Nanonavi IIs, SII NanoTechnology). Microstructures were evaluated using scanning electron microscopy (SEM; JSM$6700 \mathrm{FT}$, JEOL) operating at $5 \mathrm{kV}$. The magnetization curves were measured using the focused magneto-optical Kerr effect (MOKE; BH-PI920-HU, NEOARK) under a magnetic field of up to $1 \mathrm{kOe}$ at room temperature. The stray fields originating from the edges of the Co thin films were observed by magnetic force microscopy (MFM), in which a CoCrPt-coated cantilever was used in the AFM setup described above. The local magnetic fields generated between the two edges of the Co thin-film electrodes in SQC devices were calculated by micromagnetic simulation.

\section{RESULTS AND DISCUSSION}

Figure 1(a) shows the surface roughness as a function of the Co thin film thickness on borate glasses. For comparison, the surface roughness of Co thin films on PEN substrates, which have also been examined as magnetic thin-film electrodes of SQC devices, ${ }^{14}$ is also shown. The insets show AFM surface images of Co thin films on borate glasses and the borate glass without a Co thin film. The scanning area is $500 \times 500 \mathrm{~nm}^{2}$. Here, the surface roughness $R_{\mathrm{a}}$ is defined by $R_{a}=1 / L_{x} L_{y} \int_{0}^{L_{x}} \int_{0}^{L_{y}}|h(x, y)| d x d y$, where $h(x, y)$ is the height profile as a function of $x$ and $y$, and $L_{x(y)}$ is the lateral scanning size along the $x(y)$ direction. The surface roughness of Co thin films on borate glasses is as small as $0.44-0.65 \mathrm{~nm}$. From Fig. 1(a), it is found that this surface roughness value is smaller than that of Co thin films on PEN substrates,

(a)

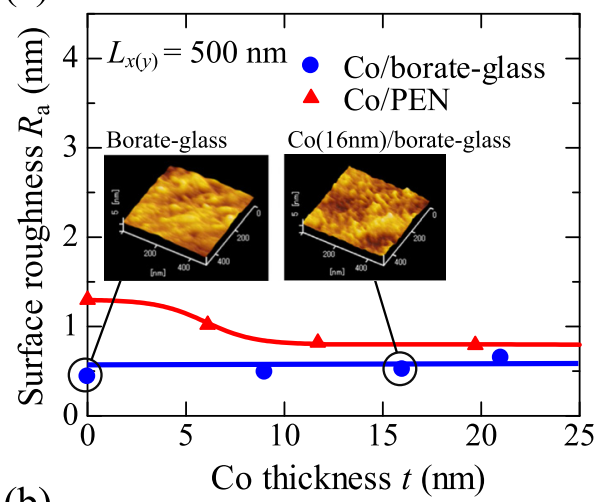

(b)

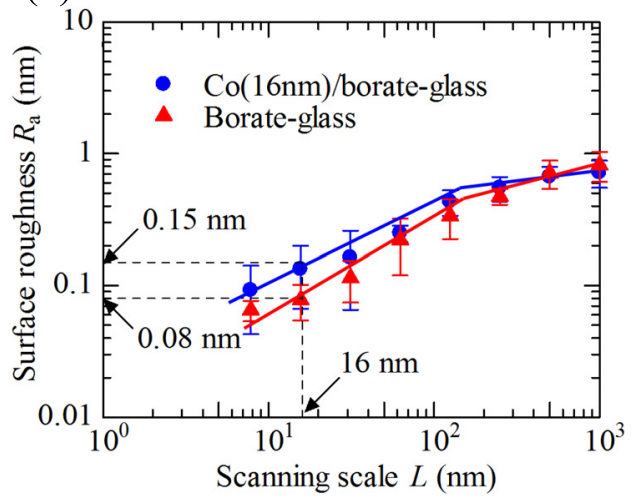

FIG. 1. (a) Surface roughness as a function of the Co thickness in Co/borateglass and Co/PEN and (b) scaling properties of the surface roughness of $\mathrm{Co}(16 \mathrm{~nm}) /$ borate-glass and borate-glass without the Co film.
$0.79-1.3 \mathrm{~nm}$. The realization of smooth Co thin films is attributed to the use of borate glass substrates with a smooth surface and a low growth rate of Co thin films. The vaporized Co atoms can move easily and freely along the in-plane direction on the borate glass substrate with a smooth surface. Under this situation, the Co atoms can diffuse on the surface for a sufficiently long time owing to the low growth rate of $0.15 \mathrm{~nm} / \mathrm{s}$. This makes it possible to form smooth surface structures, which are favored by surface energy minimization. Figure 1(b) shows the scaling properties of the surface roughness of Co thin films on borate glasses and the borate glass without the Co film. Because the junction area in SQC devices is determined by the film's thickness, the surfaces need to be smooth enough at the same scanning scale as the film thickness. As shown in Fig. 1(b), the surface roughnesses of a Co thin film with a thickness of $16 \mathrm{~nm}$ on a borate glass and that of the borate glass without the Co film are as small as $0.15 \mathrm{~nm}$ and $0.08 \mathrm{~nm}$, respectively, corresponding to less than a few atomic layers, at a scanning scale of $16 \mathrm{~nm}$. The surface roughness of a Co thin film with a thickness of less than $21 \mathrm{~nm}$ on a borate glass is less than $0.18 \mathrm{~nm}$ at the same scanning scale as the Co film thickness. These results indicate that Co thin films on borate glasses are good candidates for magnetic thin-film electrodes in SQC devices from the perspective of surface structures.

Figure 2(a) shows the magnetization curves of Co thin films on borate glasses obtained by MOKE measurements. The Co thicknesses are 8.5, 11, 15.9, and $17.5 \mathrm{~nm}$, respectively. The magnetic field is applied in the magnetic easy-

(a)

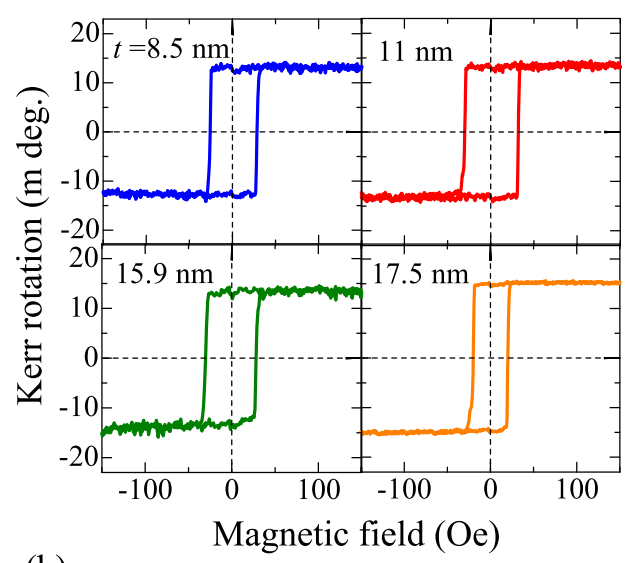

(b)

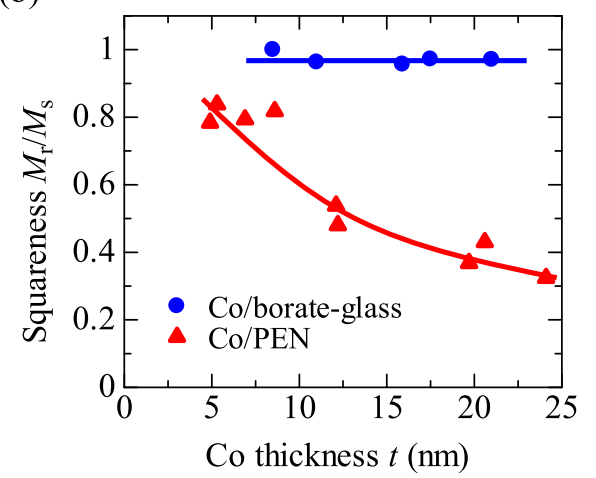

FIG. 2. (a) Magnetization curves of Co/borate-glass and (b) $M_{\mathrm{r}} / M_{\mathrm{s}}$ as a function of the Co thickness in $\mathrm{Co} /$ borate-glass and $\mathrm{Co} / \mathrm{PEN}$. 
(a)

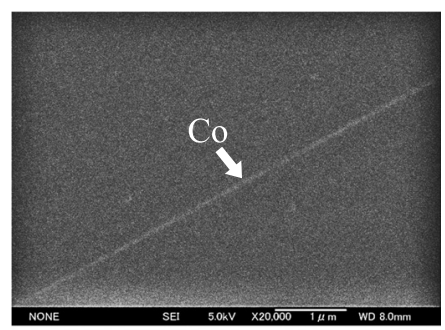

(b)

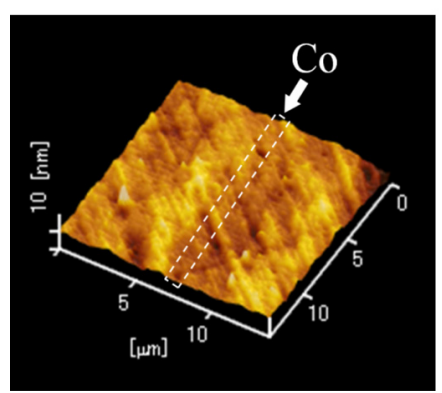

(c)

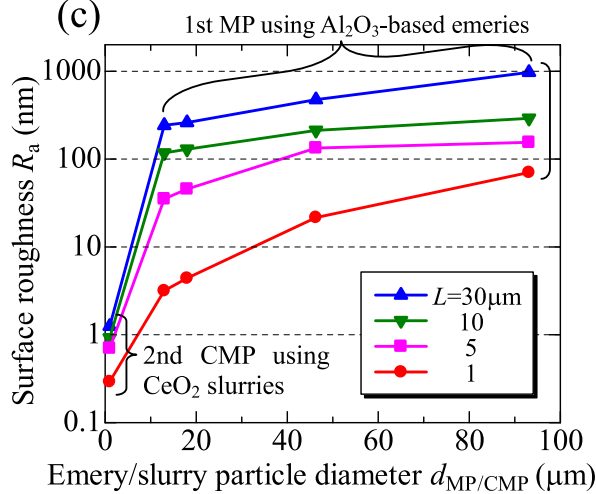

FIG. 3. (a) SEM image and (b) AFM image of the polished surfaces of borate-glass/Co/borate-glass. (c) Surface roughness of the polished surfaces of borate-glass/Co/borate-glass as a function of the particle diameter $d_{\mathrm{MP} / \mathrm{CMP}}$ of $\mathrm{Al}_{2} \mathrm{O}_{3}$-based emeries $\left(d_{\mathrm{MP}}>10 \mu \mathrm{m}\right)$ or the $\mathrm{CeO}_{2}$ slurries $\left(d_{\mathrm{CMP}}=1 \mu \mathrm{m}\right)$.

axis direction. The coercive force of a Co thin film on a borate glass is $20-30 \mathrm{Oe}$, which is almost the same as the value reported for Co films. ${ }^{15}$ The squareness of the hysteresis loop $M_{\mathrm{r}} / M_{\mathrm{s}}$, where $M_{\mathrm{r}}$ and $M_{\mathrm{s}}$ are the remanent and saturation magnetizations, respectively, is as large as 0.96-1.0. The squareness as a function of the Co thickness is shown in Fig. 2(b). The squareness of the hysteresis loop of Co thin films on borate glasses is greater than that of Co thin films on PEN substrates, 0.32-0.84. This large squareness causes a high stray field to be generated from the edges of Co thinfilm electrodes in SQC devices. These results indicate that
Co thin films on borate glasses are suitable for use as magnetic thin-film electrodes in SQC devices from the perspective of not only the surface structures but also the magnetic properties.

Figure 3 shows an SEM image of the polished surfaces of Co thin-film electrodes sandwiched between the two borate glasses. The thickness of the Co thin films is $14 \mathrm{~nm}$. From this image, Co edges can be clearly confirmed. The contrast enhancement of the Co edges is attributed to the high conductivity of Co. There is no structural difference around the Co edges, which is confirmed by the AFM observation, as shown in Fig. 3(b). As can be seen from Fig. 3(b), the Co edges are not recognized, although the Co edges exist at the center of the AFM image. This means that there is no step difference between Co thin-film electrodes and borate glasses. The roughness of the polished surfaces of Co thinfilm electrodes sandwiched between borate glasses is as small as $0.29-1.2 \mathrm{~nm}$ in a scanning area of $1 \times 1-30$ $\times 30 \mu \mathrm{m}^{2}$. The high conductivity and small surface roughness of the Co edges are realized by a three-step polishing process using MP and CMP methods, which are described below. Figure 3(c) shows the roughness of the polished surfaces of Co thin-film electrodes sandwiched between borate glasses as a function of the particle diameters $d_{\mathrm{MP}}$ (the MP process) and $d_{\mathrm{CMP}}$ (the CMP process). The $d_{\mathrm{MP}}$ of the $\mathrm{Al}_{2} \mathrm{O}_{3}$-based emeries in the first MP process is greater than $10 \mu \mathrm{m}$, and $d_{\mathrm{CMP}}$ of $\mathrm{CeO}_{2}$ slurries in the second CMP process is equal to $1 \mu \mathrm{m}$. The first MP process is performed using $\mathrm{Al}_{2} \mathrm{O}_{3}$-based emeries in the following sequence of particle diameters: 93, 46, 18, and $13 \mu \mathrm{m}$. Thereafter, the second CMP process is performed using $\mathrm{CeO}_{2}$ slurries with a particle diameter of $1 \mu \mathrm{m}$. From Fig. 3(c), it is found that the surface roughness decreases when the particle diameter of the emeries/slurries decreases and is as small as $0.29-1.2 \mathrm{~nm}$ in the second CMP process, where $\mathrm{CeO}_{2}$ slurries with a particle diameter of $1 \mu \mathrm{m}$ are used. The third CMP process is performed using $\mathrm{Al}_{2} \mathrm{O}_{3}$ slurries with a particle diameter of $0.1 \mu \mathrm{m}$. This third process causes a high increase in the conductivity of the Co edges. These established techniques for the MP and CMP methods enable the generation of a stray field from the Co edges and its observation by MFM. Figure 4 shows an MFM image of the polished surfaces of Co thin-film electrodes sandwiched between borate glasses. The thicknesses of the Co thin films are $8.5,11$, and $15 \mathrm{~nm}$, respectively. Blue colored bars in the MFM images indicate that the stray field is generated along the out-of-plane direction of the samples. As can be seen from Fig. 4, a strong (a) $t=8.5 \mathrm{~nm}$

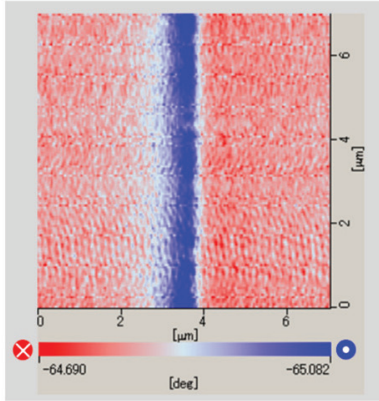

(b) $t=11 \mathrm{~nm}$

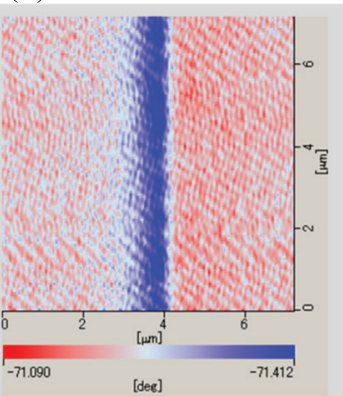

(c) $t=15 \mathrm{~nm}$

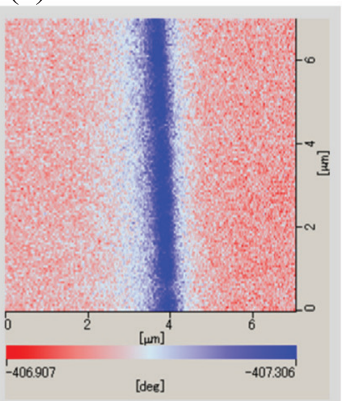

FIG. 4. MFM images of the polished surfaces of borate-glass/Co/borateglass; Co thicknesses are (a) 8.5, (b) 11 , and (c) $15 \mathrm{~nm}$, respectively. 


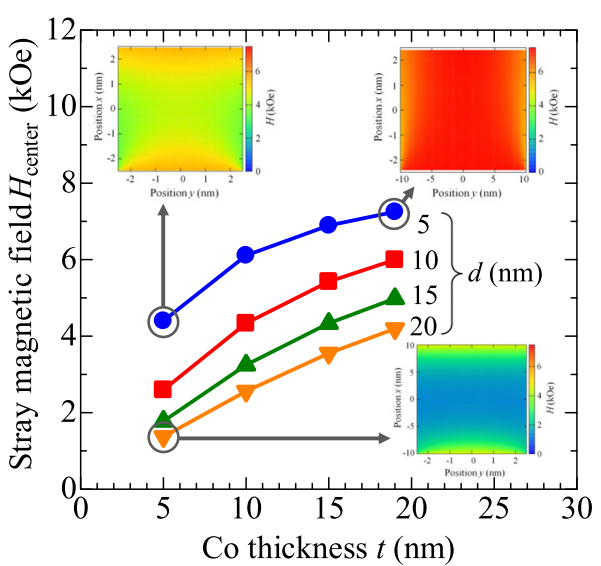

FIG. 5. Calculation results of the Co thickness dependence of the stray magnetic field between the two edges of Co thin-film electrodes in SQC devices. The insets show the distribution of the stray field; $t=5 \mathrm{~nm}$ and $d=20 \mathrm{~nm}$, $t=5 \mathrm{~nm}$ and $d=5 \mathrm{~nm}$, and $t=20 \mathrm{~nm}$ and $d=5 \mathrm{~nm}$.

stray field is generated along the out-of-plane direction from the Co edges. This behavior is consistent with the results obtained by MOKE measurements, indicating that the squareness of the hysteresis loop is as large as 0.96-1.0, as shown in Fig. 2. Here, we note that that the width of the stray field is about $0.5-1 \mu \mathrm{m}$, which is much larger than the Co thicknesses of $8.5,11$, and $18 \mathrm{~nm}$. This is attributed to a long spacing distance $(\sim 50 \mathrm{~nm})$ between the CoCrPt-coated cantilever of the MFM setup and the edge of the Co thin-film electrode, which prevents the magnetization reversal of the CoCrPt-coated cantilever. Thus, we have successfully demonstrated that Co thin-film electrodes sandwiched between borate glasses with a flat edge surface and a strong stray field generating from the edges can be fabricated using our proposed technique.

Figure 5 shows the results of calculations of the Co thickness dependence of the stray magnetic field between the two edges of the Co thin-film electrodes in SQC devices. The insets show the distribution of the stray field for varying Co thicknesses $t$ and distances between the two edges of the Co thin-film electrodes $d$; specifically, $t=5 \mathrm{~nm}, d=20 \mathrm{~nm}$; $t=5 \mathrm{~nm}, d=5 \mathrm{~nm}$; and $t=20 \mathrm{~nm}, d=5 \mathrm{~nm}$. The points $x$ and $y$ are the positions along the in-plane and out-of-plane directions of the Co films, respectively, between the two edges of the Co thin-film electrodes. The position $x=y=0 \mathrm{~nm}$ is the center of the junction between the two edges of the Co thin-film electrodes. At $t=5 \mathrm{~nm}$ and $d=20 \mathrm{~nm}$, the stray field is less than $2 \mathrm{kOe}$, and a uniform distribution is not obtained. At $t=5 \mathrm{~nm}$ and $d=5 \mathrm{~nm}$, although the stray field is beyond $4 \mathrm{kOe}$, a uniform distribution is still not obtained. At $t=20 \mathrm{~nm}$ and $d=5 \mathrm{~nm}$, the stray field measures as high as $7 \mathrm{kOe}$, and a uniform distribution is successfully obtained. From the calculation plots of Fig. 5, it is found that a high stray field beyond $6 \mathrm{kOe}$ is generated when the Co thickness is larger than $10 \mathrm{~nm}$ and the distance between the two edges of the Co thin-film electrodes is $5 \mathrm{~nm}$. This high magnetic field produces a large Zeeman effect in the sandwiched materials between the two edges of the magnetic thin-film electrodes. These experimental and calculation results conclude that SQC devices with Co thin-film electrodes sandwiched between borate glasses (Co thickness $t=10-20 \mathrm{~nm})$ can be used as spin-filter devices.

\section{ACKNOWLEDGMENTS}

This research has been partially supported by the Precursory Research for Embryonic Science and Technology program from the Japan Science and Technology Agency (JST), a Grant-in-Aid for Young Scientists from the Japan Society for the Promotion of Science (JSPS), and the Cooperative Research Program of the Network Joint Research Center for Materials and Devices from the Ministry of Education, Culture, Sports, Science and Technology (MEXT). The authors would like to express their sincere appreciation to T. Ohta and M. Takei of Hokkaido University, N. Yamashita of ISUZU GLASS CO., LTD., and T. Yamaoka of Hitachi High-Tech Science Corporation for their cooperation and helpful discussions.

${ }^{1}$ T. Miyazaki and N. Tezuka, J. Magn. Magn. Mater. 139, L231 (1995).

${ }^{2}$ J. S. Moodera, L. R. Kinder, T. M. Wong, and R. Meservey, Phys. Rev. Lett. 74, 3273 (1995).

${ }^{3}$ S. Yuasa, T. Nagahama, A. Fukushima, Y. Suzuki, and K. Ando, Nature Mater. 3, 868 (2004).

${ }^{4}$ S. S. P. Parkin, C. Kaiser, A. Panchula, P. M. Rice, B. Hughes, M. Samant, and S.-H. Yang, Nature Mater. 3, 862 (2004).

${ }^{5}$ J. S. Moodera, X. Hao, G. A. Gibson, and R. Meservey, Phys. Rev. Lett. 61, 637 (1988).

${ }^{6}$ T. Koga, J. Nitta, H. Takayanagi, and S. Datta, Phys. Rev. Lett. 88, 126601 (2002).

${ }^{7}$ A. Slobodskyy, C. Gould, T. Slobodskyy, C. R. Becker, G. Schmidt, and L. W. Molenkamp, Phys. Rev. Lett. 90, 246601 (2003).

${ }^{8}$ J. S. Moodera, T. S. Santos, and T. Nagahama, J. Phys.: Condens. Matter 19, 165202 (2007).

${ }^{9}$ T. Nagahama, T. S. Santos, and J. S. Moodera, Phys. Rev. Lett. 99, 016602 (2007).

${ }^{10}$ H. Kaiju, H. Kasa, T. Komine, T. Abe, T. Misawa, and J. Nishii, Mater. Res. Soc. Symp. Proc. 1708, vv0910 (2014).

${ }^{11}$ K. Kondo, H. Kaiju, and A. Ishibashi, J. Appl. Phys. 105, 07D522 (2009).

${ }^{12}$ H. Kaiju, K. Kondo, and A. Ishibashi, Jpn. J. Appl. Phys. Part 149 , 105203 (2010).

${ }^{13}$ H. Kaiju, K. Kondo, N. Basheer, N. Kawaguchi, S. White, A. Hirata, M. Ishimaru, Y. Hirotsu, and A. Ishibashi, Jpn. J. Appl. Phys. Part 1 51, 065202 (2012).

${ }^{14}$ H. Kaiju, T. Abe, K. Kondo, and A. Ishibashi, J. Appl. Phys. 111, 07C104 (2012).

${ }^{15}$ S. P. Li, A. Lebib, D. Peyrade, M. Natali, and Y. Chen, Appl. Phys. Lett. 77, $2743(2000)$ 